\title{
Trends in Incidence and Clinical Outcomes of Clostridioides difficile Infection, Hong Kong
}

Cosmos L.T. Guo, ${ }^{1}$ Thomas N.Y. Kwong ${ }^{1}$, Joyce W.Y. Mak, Lin Zhang, Grace C.Y. Lui, Grace L.H. Wong, Margaret Ip, Jun Yu, Joseph J.Y. Sung, William K.K. Wu, ${ }^{2}$ Sunny H. Wong ${ }^{2}$

\begin{abstract}
In support of improving patient care, this activity has been planned and implemented by Medscape, LLC and Emerging Infectious Diseases.
Medscape, LLC is jointly accredited by the Accreditation Council for Continuing Medical Education (ACCME), the Accreditation Council for Pharmacy Education (ACPE), and the American Nurses Credentialing Center (ANCC), to provide continuing education for the healthcare team.

Medscape, LLC designates this Journal-based CME activity for a maximum of 1.00 AMA PRA Category 1 Credit(s) ${ }^{\mathrm{TM}}$. Physicians should claim only the credit commensurate with the extent of their participation in the activity.

Successful completion of this CME activity, which includes participation in the evaluation component, enables the participant to earn up to 1.0 MOC points in the American Board of Internal Medicine's (ABIM) Maintenance of Certification (MOC) program. Participants will earn MOC points equivalent to the amount of CME credits claimed for the activity. It is the CME activity provider's responsibility to submit participant completion information to ACCME for the purpose of granting ABIM MOC credit.
\end{abstract}

All other clinicians completing this activity will be issued a certificate of participation. To participate in this journal CME activity: (1) review the learning objectives and author disclosures; (2) study the education content; (3) take the post-test with a $75 \%$ minimum passing score and complete the evaluation at http://www.medscape.org/journal/eid; and (4) view/print certificate. For CME questions, see page XXX.

Release date: November 18, 2021; Expiration date: November 18, 2022

Learning Objectives

Upon completion of this activity, participants will be able to:

- Describe disease burden, incidence, and clinical outcomes of CDI among hospitalized patients in Hong Kong, according to an updated territory-wide survey study

- Determine antibiotic usage and other CDI-associated risk factors and clinical outcomes among hospitalized patients in Hong Kong, according to an updated territory-wide survey study

- Identify clinical and public health implications of the epidemiologic pattern of CDI, CDI-associated risk factors, and clinical outcomes among hospitalized patients in Hong Kong, according to an updated territory-wide survey study.

\section{CME Editor}

Dana C. Dolan, BS, Copyeditor, Emerging Infectious Diseases. Disclosure: Dana C. Dolan, BS, has disclosed no relevant financial relationships.

CME Author

Laurie Barclay, MD, freelance writer and reviewer, Medscape, LLC. Disclosure: Laurie Barclay, MD, has disclosed no relevant financial relationships.

\section{Authors}

Disclosures: Cosmos L.T. Guo; Thomas N.Y. Kwong, PhD; Lin Zhang, PhD; Grace C.Y. Lui, MBChB; Jun Yu, MD, PhD; Joseph J.Y. Sung, MD, PhD; William K.K. Wu, PhD; and Sunny H. Wong, MBChB, DPhil, have disclosed no relevant financial relationships. Joyce W.Y. Mak, MBBS, MRCP(UK), has disclosed the following relevant financial relationships: received grants for clinical research from Gilead Sciences, Inc.; Janssen Pharmaceuticals, Inc. Grace L.H. Wong, MBChB (Hons), MD, has disclosed the following relevant financial relationships: served as an advisor or consultant for Gilead Sciences, Inc.; Janssen Pharmaceuticals, Inc.; served as a speaker or a member of a speakers bureau for Abbott Laboratories; AbbVie Inc.; Bristol-Myers Squibb Company; Echosens; Gilead Sciences, Inc.; received grants for clinical research from: AbbVie Inc.; Gilead Sciences, Inc. Margaret Ip, MD, has disclosed the following relevant financial relationships: served as an advisor or consultant for Cepheid; Merck Sharp \& Dohme GmbH; served as a speaker or a member of a speakers bureau for Cepheid; Merck Sharp \& Dohme GmbH; received grants for clinical research from Merck Sharp \& Dohme GmbH.

Author affiliations: The Chinese University of Hong Kong, Hong Kong, China (C.L.T. Guo, T.N.Y. Kwong, J.W.Y. Mak, L. Zhang, G.C.Y. Lui, G.L.H. Wong, M. Ip, J. Yu, J.J.Y. Sung, W.K.K. Wu, S.H. Wong); Lee Kong Chian School of Medicine, Nanyang Technological University, Singapore (J.J.Y. Sung, S.H. Wong) 
We conducted a territorywide survey to investigate the epidemiology, risk factors, and clinical outcomes of Clostridioides difficile infection (CDI) among hospitalized patients in Hong Kong. A total of 17,105 cases of CDI were identified, of which 15,717 (91.9\%) were healthcare-associated and $1,025(6.0 \%)$ were community-associated. Although CDI incidence increased substantially from 2006 to 2017, it plateaued in 2018 and 2019. The 30-day mortality rates decreased from $20.1 \%$ in 2015 to $16.8 \%$ in 2019, whereas the 60-day recurrence rates remained constant at $\approx 11 \%$ during the study period. Cross-correlation statistic showed significant correlations between incidence trend and overall antimicrobial drug use ( $r=0.865, p<0.001)$, which has decreased as a result of an antibiotic stewardship program initiated in 2017. Our data suggest a turning point in $C$. difficile epidemiology that could be related to the changing pattern of antimicrobial drug use.

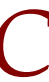

lostridioides difficile infection (CDI) is a common nosocomial disease; symptoms range from mild diarrhea to life-threatening colitis and toxic megacolon. CDI is associated with a high mortality rate, particularly for patients $>75$ years of age (1). Epidemiologic studies have identified its substantial incidence especially in the United States and in many countries in Europe $(2,3)$. Recent data suggested that its overall incidence in some of these countries have reached a plateau. For instance, the US Centers for Disease Control and Prevention (CDC) reported a decrease in CDI incidence from 2014 to 2017 (2), whereas the overall CDI incidence in Sweden has decreased by $22 \%$ from 2012 to 2016 (4). These declines are often attributed to the implementation of antibiotic stewardship programs. Nonetheless, community-acquired CDI (CACDI) represents a growing threat; incidence of CACDI doubled from 2011-2015 (5).

The epidemiologic patterns in different geographic regions are highly dynamic. Outbreaks of CDI in North America and Europe were once predominantly caused by the $C$. difficile ribotype 027 (6), which was rarely reported about in Asia (7). Instead, C. difficile ribotype 017 has been the predominant strain in Asia (8). Other toxigenic strains, such as $C$. difficile ribotype 369 , which was associated with multiple epidemics in Japan, have been reported in various Southeast Asia countries (9), whereas $C$. difficile ribotype 002 was reported to be common in Hong Kong, China, and was associated with increased virulence $(10,11)$. Continuous surveillance, therefore, is important to prevent outbreaks of CDI. However, epidemiologic data of CDI in Asia remain sparse. We have previously characterized the molecular and antimicrobial susceptibility patterns of prevalent $C$. difficile ribotypes in Hong
Kong (12). We also conducted an observational study to investigate CDI disease burden and clinical outcomes among hospitalized patients in Hong Kong, which showed a rapidly increasing incidence until 2014 (13). In this study, we continued to update the epidemiologic pattern of CDI among hospitalized patients in Hong Kong and characterize CDI-associated risk factors and clinical outcomes.

We conducted this study in accordance with the Declaration of Helsinki (2013 version). The Joint Clinical Research Ethics Committee of the Chinese University of Hong Kong and Hospital Authority New Territory East Cluster approved the study. All clinical data were anonymized by the Clinical Data Analysis and Reporting System (CDARS), and all potential identifiers were removed upon return of database searches.

\section{Methods}

\section{Study Population and Data Extraction}

We identified digital records of all patients hospitalized in public hospitals with a laboratory-confirmed diagnosis of CDI in Hong Kong during January 1, 2015-December 31, 2019, from CDARS, a database of public hospital patient records managed by the Hong Kong Hospital Authority. We obtained clinical data including the patient demographics, laboratory results, drug prescription records, clinical outcomes, and diagnoses of underlying conditions. Patient demographic data include age and gender. We identified relevant diagnoses using codes from the International Classification of Diseases, 9th Revision, in accordance with the Charlson Comorbidity Index (14). We also obtained data on antimicrobial drug use and other drug use within 8 weeks before CDI diagnosis.

\section{Case Identification and Definitions}

We defined a CDI case as positive result obtained from culture, toxin, or molecular assay for a diarrheal fecal specimen collected from inpatient residents $\geq 18$ years of age. As described previously (13), patients with samples obtained $>48$ hours after admission or those who were hospitalized in a healthcare facility within the previous 4 weeks were classified as cases of healthcare-associated CDI (HA-CDI). We defined community-associated CDI (CA-CDI) as patients who had not been hospitalized in a healthcare facility within the previous 12 weeks. We defined patients who had been hospitalized in a healthcare facility within the previous $4-12$ weeks as indeterminate. We classified patients with a maximum leukocyte count 
$>15,000$ cells $/ \mu \mathrm{L}$ or $>50 \%$ increase in serum creatinine level as having cases of severe CDI, as defined by the Infectious Diseases Society of America (15). We defined refractory disease as a nonresponding disease requiring $>14$ days of continued treatment and a treatment period as a period during which records of drug prescription records indicate continuous antimicrobial treatment with $\leq 3$ days of interruption.

\section{Antimicrobial Drug Use Data}

Because the use of antimicrobial drugs is a major risk factor for CDI, we extracted from the data the overall corporate use of antimicrobial drugs in public hospitals in Hong Kong and analyzed. The data were recorded as daily defined doses (DDDs), which is the assumed average maintenance dose per day for each drug. These can demonstrate the absolute changes in use, as well as DDDs per 1,000 bed-days occupied (DDD/1,000 BDO), which can demonstrate changes in use relative to hospital occupancy. Broad-spectrum antimicrobial drugs include cefepime, ceftazidime, cefotaxime, cefoperazone/sulbactam, piperacillin, piperacillin/tazobactam, carbapenems, and quinolones. We determined the risk for CDI for each drug class on the basis of its association with CDI (1). Highrisk drugs were lincosamides, cephalosporins, fluoroquinolones, amoxicillin, and ampicillin. Medium-risk drugs included sulphonamides and macrolides. Lowrisk drugs included tetracyclines (1).

\section{Statistical Analysis}

We analyzed data with $\mathrm{R}$ version 3.6.0. ( $\mathrm{R}$ Foundation for Statistical Computing, https://www.rproject.org) We defined annual crude incidence of CDI as the number of patients given a diagnosis of CDI per 100,000 adult population, using data obtained from the Hong Kong Census and Statistics Department. We analyzed potential predictors for 30 -day mortality rate and 60 -day recurrence rate using univariate and multivariate forward Wald logistic regression. We used Cox proportional hazard regression to identify factors that decreased the time to recurrence after an episode. We used $X^{2}$ test of proportion to compare differences in incidences, mortality rates, and recurrence rates. We used crosscorrelation to identify correlation between CDI incidence and antimicrobial drug use and $p=0.05$ as a measure of statistical significance.

\section{Results}

\section{Disease Burden and Incidence}

During 2015-2019, we identified 17,105 cases of CDI among hospitalized patients in Hong Kong (average 3,421 cases/year). Of these cases, 15,717 (91.9\%) were HA-CDI and 1,025 (6.0\%) were CA-CDI. The remaining 363 cases $(2.1 \%)$ were indeterminate (Table 1; Appendix Figure 1).

Although a rapid increase of CDI incidence was observed during 2006-2014, the crude incidence of CDI in Hong Kong remained relatively stable and the average annual percentage change (APC) from 2015 to 2019 showed a modest increase of $1.53 \%$ (Table 1; Figure, panel A). Among the different age groups, the CDI incidence showed a significant decrease for patients $\geq 75$ years of age (Figure, panel B). We observed a similar downward trend in the overall incidences of HA-CDI (Figure, panel A), the first time since the start of our previous study in 2006 (13). However, this decrease was not evident in the younger patient groups between 2015 and 2019, despite these groups only representing a minor proportion of CDI incidence (Table 1; Figure).

The median age of patients was 77 years (interquartile range [IQR] 63-86 years); $51.8 \%$ were female and $48.2 \%$ male (Table 2). The number of patients from old-age homes has significantly decreased, from $29.2 \%$ in 2015 to $22.8 \%$ in 2019 ( $p<0.001$ ) (Table $3)$, compared with an average of $30.2 \%$ in the period 2006-2014. Most patients have taken high-risk antimicrobial drugs $(81.4 \%)$, broad-spectrum antimicrobial drugs $(59.3 \%)$, or proton-pump inhibitors (PPI) $(62.1 \%)$ within 8 weeks before diagnosis of CDI (Table 2). Of note, during the period of 2015-2019, the proportion of severe CDI has decreased from $38.2 \%$ to $31.2 \%$ of patients $(\mathrm{p}<0.001)$ (Table 3$)$.

\begin{tabular}{|c|c|c|c|c|c|c|c|}
\hline \multirow[b]{2}{*}{ Year } & \multirow[b]{2}{*}{ Adult population } & \multicolumn{3}{|c|}{ No. cases } & \multicolumn{3}{|c|}{ Incidence† } \\
\hline & & Overall & HA-CDI & CA-CDI & Overall & HA-CDI & CA-CDI \\
\hline 2015 & $6,247,460$ & 3,160 & 2,921 & 181 & 50.6 & 46.8 & 2.9 \\
\hline 2016 & $6,301,560$ & 3,303 & 3,058 & 185 & 52.4 & 48.5 & 2.9 \\
\hline 2017 & $6,357,420$ & 3,618 & 3,303 & 231 & 56.9 & 52.0 & 3.6 \\
\hline 2018 & $6,410,080$ & 3,557 & 3,248 & 223 & 55.5 & 50.7 & 3.5 \\
\hline 2019 & $6,481,000$ & 3,467 & 3,187 & 205 & 53.5 & 49.2 & 3.2 \\
\hline
\end{tabular}

${ }^{*}$ Adult is defined as a person $>18$ years of age. CA-CDI, community-associated Clostridioides difficile infection; HA-CDI, healthcare-associated Clostridioides difficile infection.

†No. cases $/ 100,000$ adults. 

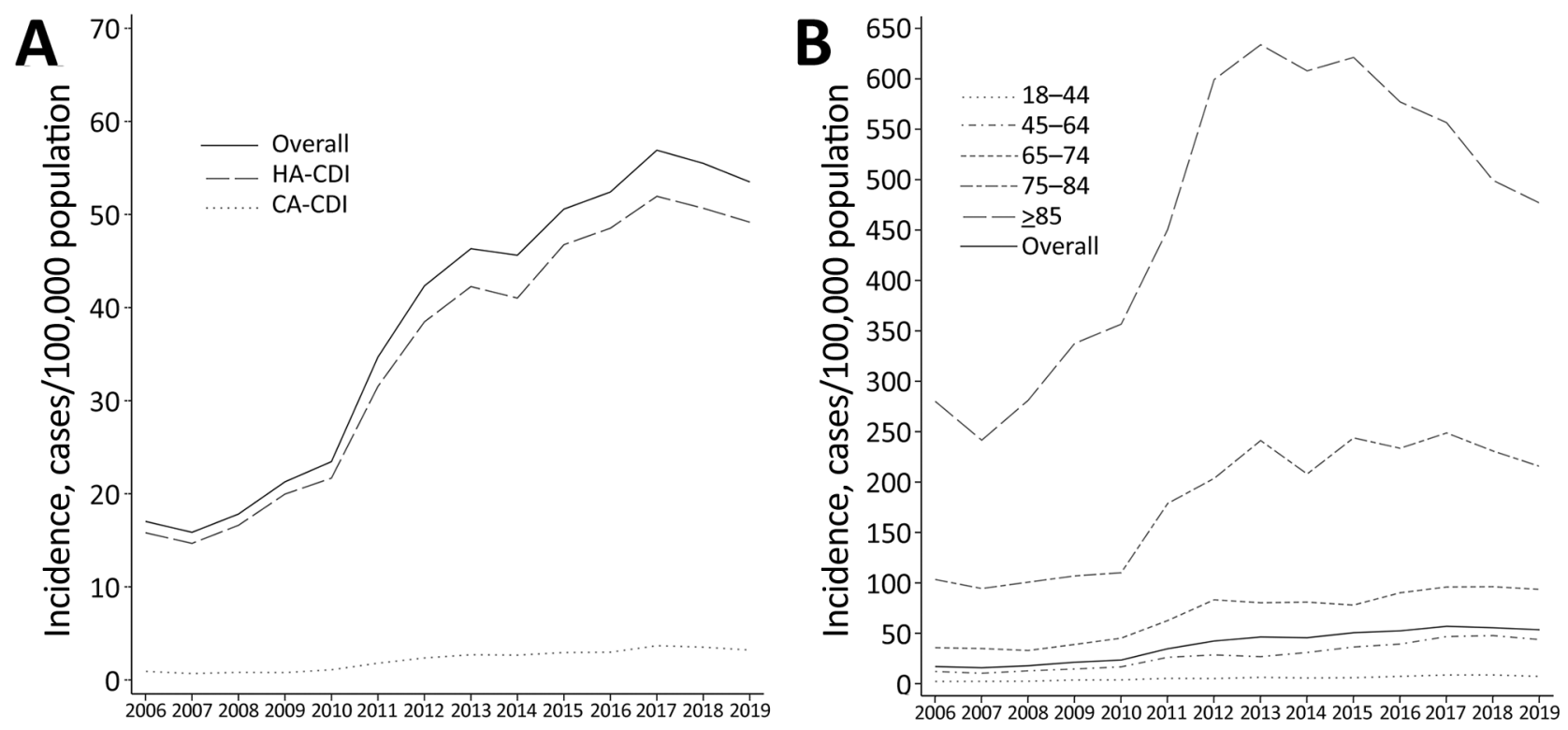

Figure. Clostridioides difficile infections in adults, Hong Kong, 2006-2019. Data for 2006-2014 were acquired from a previous study (13). A) Crude incidence of healthcare-associated and community-associated C. difficile infections. B) Incidence of infections by age group.

\section{Clinical Outcomes and Risk Factors}

The 30-day all-cause mortality rates have decreased from $20.1 \%$ in 2015 to $16.8 \%$ in 2019 ( $p=0.002$ ), substantially lower than the previous decrease of $22.5 \%$ during the period 2006-2014 (Appendix Table 1) (13). Multivariate logistic regression analysis indicated that the main predictors for death in 30 days were advanced age (>85 years, adjusted OR [aOR] 7.23, 95\% CI 5.29-10.12; 75-84 years, aOR 4.30, 95\% CI 3.16-5.98) and metastatic tumor (aOR 2.63, 95\% CI 2.21-3.12) (Table 3).

The 60-day recurrence rate remained at $\approx 11 \%$ (Appendix Table 1). Cox regression analysis showed that the main predictors of 60-day recurrence were healthcare-associated CDI (adjusted hazard ratio [aHR] 8.15, 95\% CI 5.25-12.63) and use of quinolones (aHR 1.59, 95\% CI 1.41-1.78) or broad-spectrum antibiotics (aHR 1.37, 95\% CI 1.18-1.59) within 8 weeks before diagnosis of CDI (Appendix Table 2). Refractory disease rates decreased from $13.6 \%$ in 2015 to $11.3 \%$ in 2019 , but the change was not statistically significant $(\mathrm{p}<0.076)$.

The number of patients who used tetracyclines within 8 weeks before CDI increased from $2.7 \%$ in 2015 to $6.2 \%$ in 2019 ( $\mathrm{p}<0.001)$. In contrast, patient exposure to certain known risk factors has decreased during the period, including the use of $\mathrm{H} 2$ antagonists (from $35.2 \%$ to $25.0 \%$; $<<0.001$ ), high-risk antibiotics (from $85.5 \%$ to $77.7 \%$; $p<0.001$ ), penicillin group of drugs (from $74.2 \%$ to $68.5 \%$; $<<0.001$ ) and fluoroquinolones (from $27.4 \%$ to $20.7 \%$; $<<0.001$ ).
The risk factor of having cerebrovascular accident as an underlying condition also decreased in these patients (from $21.9 \%$ to $15.9 \%$; p<0.001) (Appendix Table 3).

\section{Comparison of HA-CDI and CA-CDI}

The proportion of HA-CDI among all patients has remained at $\approx 90 \%$ and of CA-CDI at $\approx 5 \%$ (Appendix Figure). The year-to-year changes for the CA-CDI and HA-CDI incidence rates were not statistically significant, suggesting a static trend during the period (Table 1). Comparisons between the patients showed that HA-CDI patients had a median age of 77 (IQR 63-86) years, versus a median age of 74 (IQR 58-85) years for CA-CDI patients. Significantly more HA-CDI patients had severe CDI $(36.5 \%$ vs $29.0 \%$; $\mathrm{p}<0.001)$ and underlying conditions compared with CA-CDI patients (Table 2). HA-CDI patients were more likely to have exposure to high-risk antimicrobial drugs $(86.0 \%$ vs. $26.5 \%$; $<<0.001)$ and broadspectrum antimicrobials $(63.5 \%$ vs $9.2 \% ; \mathrm{p}<0.001)$ within 8 weeks before CDI. The 30-day mortality rate was $19.7 \%$ for HA-CDI but $8.5 \%$ for CA-CDI patients $(p<0.001)$, although both rates have decreased compared with the earlier period of 2006-2014 (13). Although the 30-day mortality rate for HA-CDI decreased from 2015 to 2019, we did not observe an obvious trend in mortality rate for CA-CDI patients $(p=0.354)$ (Appendix Table 1$)$. The 60-day recurrence rate was $12.4 \%$ for HA-CDI, whereas none of the CA-CDI patients had a recurrence (Table 2). 


\section{Data on Antimicrobial Drug Use}

The overall use of antimicrobial drugs per year, measured as DDD/1,000 BDO, increased from 1,206 in 2006 to 4,747 in 2018 but then decreased to 3,968 in 2019 (Appendix Table 4). Annual use shared a significant correlation $(r=0.865 ; p<0.001)$ with the CDI incidence. In terms of $\mathrm{DDD} / 1,000$ $\mathrm{BDO}$, we observed the highest levels of correlation for lincosamides $(r=0.907 ; p<0.001)$, carbapenems $(\mathrm{r}=0.893 ; \mathrm{p}<0.001)$ and sulphonamides $(\mathrm{r}=0.872$;

\begin{tabular}{|c|c|c|c|c|}
\hline Characteristic & Overall & CA-CDI & HA-CDI & Indeterminate \\
\hline All patients & 17,105 & $1,025(6.0)$ & $15,717(91.9)$ & $363(2.1)$ \\
\hline \multicolumn{5}{|l|}{ Age, y } \\
\hline Median (IQR) & $77(63-86)$ & $74(58-85)$ & $77(63-86)$ & $78(62-86)$ \\
\hline$<44$ & $1,056(6.2)$ & $138(13.5)$ & $901(5.7)$ & $17(4.7)$ \\
\hline $45-64$ & $3,679(21.5)$ & $221(21.6)$ & $3,375(21.5)$ & 83 (22.9) \\
\hline $65-74$ & $3,027(17.7)$ & $173(16.9)$ & $2,793(17.8)$ & $61(16.8)$ \\
\hline $75-84$ & $4,340(25.4)$ & $207(20.2)$ & $4,044(25.7)$ & $89(24.5)$ \\
\hline$\geq 85$ & $5,003(29.2)$ & $286(27.9)$ & 4,604 (29.3) & $113(31.1)$ \\
\hline \multicolumn{5}{|l|}{ Sex } \\
\hline M & $8,252(48.2)$ & $442(43.1)$ & $7,642(48.6)$ & 168 (46.3) \\
\hline $\mathrm{F}$ & $8,853(51.8)$ & $583(56.9)$ & $8,075(51.4)$ & $195(53.7)$ \\
\hline Admission from $\mathrm{OAH}$ & $4,321(25.3)$ & $209(20.4)$ & $4,003(25.5)$ & $109(30.0)$ \\
\hline IDSA-defined severe disease & $5,871(35.8)$ & $295(29.0)$ & $5,482(36.5)$ & $94(26.2)$ \\
\hline \multicolumn{5}{|l|}{ Diagnostic test } \\
\hline Bacterial culture & $8,191(40.9)$ & $468(39.4)$ & $7,560(41.0)$ & $163(38.6)$ \\
\hline Nucleic acid amplification test & $8,994(44.9)$ & $547(46.0)$ & $8,261(44.8)$ & $186(44.1)$ \\
\hline Toxin detection & $2,855(14.2)$ & $173(14.6)$ & $2,609(14.2)$ & $73(17.3)$ \\
\hline \multicolumn{5}{|l|}{ Antimicrobial drug use } \\
\hline High-risk drugs & $13,932(81.4)$ & $272(26.5)$ & $13,519(86.0)$ & $141(38.8)$ \\
\hline Medium-risk drugs & $1,562(9.1)$ & $23(2.2)$ & $1,526(9.7)$ & $13(3.6)$ \\
\hline Low-risk drugs & $4,286(25.1)$ & $17(1.7)$ & $4,257(27.1)$ & $12(3.3)$ \\
\hline Broad-spectrum drugs & $10,147(59.3)$ & $94(9.2)$ & $9,986(63.5)$ & $67(18.5)$ \\
\hline \multicolumn{5}{|l|}{ Use of other drugs } \\
\hline Proton pump inhibitor & $10,614(62.1)$ & $201(19.6)$ & $10,255(65.2)$ & $158(43.5)$ \\
\hline $\mathrm{H} 2$ antagonist & $4,950(28.9)$ & $158(15.4)$ & $4,722(30.0)$ & $70(19.3)$ \\
\hline Corticosteroid & $4,477(26.2)$ & $81(7.9)$ & $4,350(27.7)$ & $46(12.7)$ \\
\hline \multicolumn{5}{|l|}{ Underlying conditions } \\
\hline Myocardial infarction & $1,212(7.1)$ & $37(3.6)$ & $1,144(7.3)$ & $31(8.5)$ \\
\hline Congestive heart failure & $2,407(14.1)$ & $64(6.2)$ & $2,292(14.6)$ & $51(14.0)$ \\
\hline Peripheral vascular disease & $556(3.3)$ & $10(1.0)$ & $536(3.4)$ & $10(2.8)$ \\
\hline Cerebrovascular disease & $3,051(17.8)$ & 91 (8.9) & $2,887(18.4)$ & $73(20.1)$ \\
\hline Chronic pulmonary disease & $1,937(11.3)$ & $84(8.2)$ & $1,806(11.5)$ & $47(12.9)$ \\
\hline Mild liver disease & $338(2.0)$ & $18(1.8)$ & $301(1.9)$ & $19(5.2)$ \\
\hline Severe liver disease & $243(1.4)$ & $11(1.1)$ & $221(1.4)$ & $11(3.0)$ \\
\hline Diabetes mellitus & $3,624(21.2)$ & $131(12.8)$ & $3,414(21.7)$ & $79(21.8)$ \\
\hline Diabetes mellitus with complications & $1,492(8.7)$ & $44(4.3)$ & $1,406(8.9)$ & $42(11.6)$ \\
\hline Moderate/severe kidney disease & $3,363(19.7)$ & $94(9.2)$ & $3,178(20.2)$ & $91(25.1)$ \\
\hline Nonmetastatic cancer & 3,403 (19.9) & $75(7.3)$ & $3,268(20.8)$ & $60(16.5)$ \\
\hline Metastatic cancer & $970(5.7)$ & $21(2.0)$ & $932(5.9)$ & $17(4.7)$ \\
\hline HIV & $16(0.1)$ & $1(0.1)$ & $15(0.1)$ & 0 \\
\hline Paraplegia & $356(2.1)$ & $12(1.2)$ & $336(2.1)$ & $8(2.2)$ \\
\hline Connective tissue disease & $174(1.0)$ & $15(1.5)$ & $152(1.0)$ & 7 (1.9) \\
\hline Dementia & $863(5.0)$ & $47(4.6)$ & $793(5.0)$ & $23(6.3)$ \\
\hline Peptic ulcer & $867(5.1)$ & $24(2.3)$ & $826(5.3)$ & $17(4.7)$ \\
\hline \multicolumn{5}{|l|}{ Outcomes } \\
\hline Episode death & $3,220(18.8)$ & $73(7.1)$ & $3,117(19.8)$ & $30(8.3)$ \\
\hline 30-day mortality & $3,225(18.9)$ & $87(8.5)$ & $3,100(19.7)$ & 38 (10.5) \\
\hline 60-day mortality & $4,738(27.7)$ & $117(11.4)$ & $4,562(29.0)$ & $59(16.3)$ \\
\hline 30-day recurrence & 1,968 (11.5) & 0 & $1,947(12.4)$ & $21(5.8)$ \\
\hline Refractory disease & $2,155(12.6)$ & $59(5.8)$ & $2,064(13.1)$ & $32(8.8)$ \\
\hline \multicolumn{5}{|l|}{ Procedures } \\
\hline Partial colectomy & $3(0.0)$ & $1(0.1)$ & $2(0.0)$ & 0 \\
\hline Left colectomy & $3(0.0)$ & 0 & $3(0.0)$ & 0 \\
\hline Right colectomy & $6(0.0)$ & 0 & $6(0.0)$ & 0 \\
\hline Sigmoid colectomy & $1(0.0)$ & 0 & $1(0.0)$ & 0 \\
\hline Total colectomy & $4(0.0)$ & $1(0.1)$ & $3(0.0)$ & 0 \\
\hline Fecal microbiota transplant & $3(0.0)$ & 0 & $3(0.0)$ & 0 \\
\hline
\end{tabular}

*Values are no. (\%) patients except as indicated. CA-CDI, community-associated Clostridioides difficile infection; HA-CDI, healthcare-associated

Clostridioides difficile infection; IDSA, Infectious Diseases Society of America; IQR, interquartile range; OAH, old age home. 
Trends in C. difficile Infection, Hong Kong

Table 3. Logistic regression analysis of potential independent variables associated with 30-day mortality for Clostridioides difficile infection, Hong Kong, China, 2015-2019*

\begin{tabular}{|c|c|c|c|c|}
\hline \multirow[b]{2}{*}{ Variable } & \multicolumn{2}{|c|}{ Univariate } & \multicolumn{2}{|c|}{ Multivariate } \\
\hline & OR $(95 \% \mathrm{Cl})$ & $p$ value & OR $(95 \% \mathrm{Cl})$ & $p$ value \\
\hline \multicolumn{5}{|l|}{ Age, y } \\
\hline$<44$ & Referent & NA & Referent & NA \\
\hline $45-64$ & $3.115(2.301-4.322)$ & $<0.001$ & $2.458(1.794-3.449)$ & $<0.001$ \\
\hline $65-74$ & 4.203 (3.105-5.829) & $<0.001$ & $3.203(2.334-4.502)$ & $<0.001$ \\
\hline $75-84$ & $6.237(4.643-8.595)$ & $<0.001$ & $5.384(3.944-7.531)$ & $<0.001$ \\
\hline$\geq 85$ & 7.986 (5.959-10.986) & $<0.001$ & $7.633(5.583-10.70)$ & $<0.001$ \\
\hline Male sex & $1.124(1.041-1.213)$ & 0.0029 & $1.221(1.121-1.330)$ & $<0.001$ \\
\hline IDSA-defined severe disease & $2.296(2.121-2.486)$ & $<0.001$ & $2.159(1.986-2.347)$ & $<0.001$ \\
\hline Healthcare-associated disease & $2.483(2.066-3.010)$ & $<0.001$ & $1.378(1.119-1.708)$ & 0.003 \\
\hline Admission from $\mathrm{OAH}$ & $1.716(1.579-1.864)$ & $<0.001$ & $1.327(1.203-1.463)$ & $<0.001$ \\
\hline \multicolumn{5}{|l|}{ Diagnostic test } \\
\hline Bacterial culture & Referent & NA & Referent & NA \\
\hline Nucleic acid amplification test & $1.070(0.991-1.157)$ & 0.0845 & $1.046(0.962-1.138)$ & 0.294 \\
\hline Toxin detection & $0.995(0.669-1.438)$ & 0.9799 & $0.904(0.592-1.343)$ & 0.629 \\
\hline \multicolumn{5}{|l|}{ Antimicrobial drug use } \\
\hline High-risk drugs & $2.243(1.990-2.534)$ & $<0.001$ & $0.753(0.603-0.939)$ & 0.012 \\
\hline Medium-risk drugs & $1.021(0.893-1.164)$ & 0.7602 & $1.988(0.653-8.670)$ & 0.281 \\
\hline Low-risk drugs & $1.193(1.094-1.300)$ & 0.0001 & $0.841(0.647-1.092)$ & 0.193 \\
\hline Broad-spectrum drugs & $1.673(1.542-1.817)$ & $<0.001$ & $1.402(1.244-1.581)$ & $<0.001$ \\
\hline Aminoglycosides & $0.907(0.773-1.059)$ & 0.2223 & $1.026(0.825-1.272)$ & 0.815 \\
\hline Beta-lactamase inhibitor & $2.276(2.063-2.516)$ & $<0.001$ & $1.465(1.047-2.052)$ & 0.026 \\
\hline Carbapenem & $1.229(1.118-1.349)$ & $<0.001$ & $1.233(0.967-1.571)$ & 0.091 \\
\hline Cephalosporin & $1.180(1.087-1.279)$ & 0.0001 & $0.936(0.848-1.033)$ & 0.187 \\
\hline Lincosamides & $0.901(0.554-1.400)$ & 0.6580 & $1.126(0.674-1.800)$ & 0.634 \\
\hline Macrolides & $1.411(1.201-1.650)$ & $<0.001$ & $0.604(0.140-1.812)$ & 0.425 \\
\hline Penicillin & $2.208(2.003-2.438)$ & $<0.001$ & $1.165(0.840-1.624)$ & 0.362 \\
\hline Quinolones & $1.115(1.020-1.218)$ & 0.0161 & $0.939(0.843-1.045)$ & 0.251 \\
\hline Sulphonamides & $0.588(0.465-0.734)$ & $<0.001$ & $0.395(0.092-1.167)$ & 0.138 \\
\hline Tetracyclines & $1.291(1.081-1.534)$ & 0.0043 & $1.219(0.955-1.548)$ & 0.108 \\
\hline \multicolumn{5}{|l|}{ Use of other drugs } \\
\hline Proton pump inhibitor & $1.614(1.486-1.755)$ & $<0.001$ & $1.182(1.073-1.304)$ & $<0.001$ \\
\hline $\mathrm{H} 2$ antagonist & $1.237(1.136-1.346)$ & $<0.001$ & $1.225(1.109-1.353)$ & $<0.001$ \\
\hline Corticosteroid & $0.954(0.876-1.038)$ & 0.2759 & $0.919(0.835-1.010)$ & 0.080 \\
\hline \multicolumn{5}{|l|}{ Underlying conditions } \\
\hline Myocardial infarction & $1.268(1.100-1.457)$ & 0.0009 & $1.022(0.871-1.195)$ & 0.791 \\
\hline Congestive heart failure & $1.632(1.475-1.804)$ & $<0.001$ & $1.358(1.208-1.525)$ & $<0.001$ \\
\hline Peripheral vascular disease & $1.218(0.989-1.489)$ & 0.0588 & $0.980(0.781-1.221)$ & 0.859 \\
\hline Cerebrovascular disease & 1.204 (1.092-1.325) & 0.0002 & $1.021(0.911-1.143)$ & 0.722 \\
\hline Nonmetastatic cancer & 1.498 (1.368-1.638) & $<0.001$ & $1.657(1.468-1.869)$ & $<0.001$ \\
\hline Metastatic cancer & 2.827 (2.466-3.237) & $<0.001$ & $2.627(2.209-3.124)$ & $<0.001$ \\
\hline Diabetes mellitus & $1.267(1.157-1.386)$ & $<0.001$ & $1.052(0.948-1.168)$ & 0.339 \\
\hline Diabetes mellitus with complications & $1.226(1.076-1.394)$ & 0.0020 & $1.205(1.029-1.409)$ & 0.020 \\
\hline Mild liver disease & $1.005(0.758-1.314)$ & 0.9694 & $1.192(0.829-1.690)$ & 0.333 \\
\hline Severe liver disease & $1.204(0.878-1.623)$ & 0.2360 & $1.643(1.090-2.451)$ & 0.016 \\
\hline Peptic ulcer & $1.283(1.087-1.508)$ & 0.0029 & $1.060(0.885-1.264)$ & 0.523 \\
\hline Chronic pulmonary disease & $1.302(1.161-1.459)$ & $<0.001$ & $1.006(0.883-1.144)$ & 0.926 \\
\hline Moderate/severe kidney disease & $1.203(1.095-1.320)$ & 0.0001 & $1.383(1.231-1.553)$ & $<0.001$ \\
\hline Connective tissue disease & $0.556(0.338-0.865)$ & 0.0139 & $0.970(0.573-1.563)$ & 0.904 \\
\hline Paraplegia & $1.152(0.885-1.482)$ & 0.2810 & $1.054(0.779-1.410)$ & 0.726 \\
\hline Dementia & $1.364(1.158-1.600)$ & 0.0002 & $1.033(0.864-1.231)$ & 0.718 \\
\hline HIV & $0.287(0.016-1.415)$ & 0.2253 & $0.562(0.030-3.080)$ & 0.590 \\
\hline
\end{tabular}

$\mathrm{p}<0.001)$, followed by penicillin $(\mathrm{r}=0.847 ; \mathrm{p}<0.001)$ and quinolones $(r=0.825 ; p<0.001)$. Similar to the trend in overall antimicrobial drug use, all of these drugs had a decrease in use in 2019, after consistent increases from 2006-2018. We grouped ampicillin and amoxicillin together with other penicillin group drugs because their combined use attributed to $80 \%$ of all penicillin use from 2006-2019. In the same period, we observed increased use of tetracyclines, from $9.22 \mathrm{DDD} / 1,000 \mathrm{BDO}$ in 2006 to 193.44 DDD/1,000 BDO in 2019.

\section{Discussion}

In this study, we investigated the latest disease burden of CDI in Hong Kong to provide a complete picture of continual disease surveillance since 2006. Because the public hospitals provide $>90 \%$ of inpatient medical service in Hong Kong, this study provides a 
comprehensive and near-complete data on the disease epidemiology among hospitalized patients in the territory. Our main finding was a decrease in the incidence of CDI in 2018 and 2019, in contrast with the distinctive increasing trend in 2006-2017 (13). The average APC during 2015-2019 showed a 1.53\% increase, in contrast with the $13.76 \%$ increase for the average APC from 2006-2014. Year-to-year changes of crude CDI incidence in 2015-2019, except for 20162017, were statistically insignificant, suggesting that the incidence might have reached a plateau. Our reported incidence in Hong Kong (56.9 cases/100,000 population in 2017) was higher than incidence in the United Kingdom (24 cases/100,000 population in 2017), where CDI incidence has seen a decrease that was mostly attributed to a successful antimicrobial drug stewardship program $(16,17)$. In comparison, Guh et al. reported an estimated crude incidence of 143.6 cases/100,000 population in the United States for 2017 (2). Despite the decrease in CDI incidence since 2011, it is still more than double the incidence in Hong Kong (18). In contrast, Liao et al. reported an overall crude CDI incidence in China during 20092016 was 34 cases/100,000 population, a relatively low value compared with our observations (19).

The trends in CDI incidence from 2006-2019 may partially be explained by changes in antimicrobial drug use. Usage rates of many drugs, such as penicillin, lincosamides (including clindamycin), quinolones, sulphonamides, and carbapenems have demonstrated significant correlations with CDI incidence. Penicillin, lincosamides, and quinolones are known to be high-risk for CDI, whereas sulphonamides and carbapenems carry a medium to low risk (1). The changes in penicillin use are likely the most relevant, because they were the most prescribed class of drugs during this period. We saw in the same period increased use of tetracyclines, which have been repeatedly demonstrated to have a relatively low risk for CDI (20). The changes in antimicrobial drug use may be attributable to Hong Kong's antibiotic stewardship program, which was updated in 2017 (21). These changes are consistent with our observation on the decreased use of antimicrobial drugs, including the penicillin group, fluoroquinolones, and other high-risk drugs, in CDI patients from 2015-2019. By extrapolating the CDI incidence from 2006-2017 to predict incidence in 2018-2019, we observed that the predicted incidence in 2019 would be $20 \%$ higher than the actual incidence, showing that the potential effect antibiotic stewardship had on the status quo.

In addition to a decrease in incidence, we observed a decrease in 30-day mortality rates, for which there are myriad plausible explanations. The decrease may be attributed to improved effectiveness of treatment and management efforts for CDI, successful antibiotic stewardship programs, or increased use of fecal microbiota transplant as a treatment (22). Alternatively, a decrease in deaths may be attributable to a change in prevalence of ribotypes or their virulence, such as ribotype 002, which is common in Hong Kong. This difference may also explain the decrease in the relative proportion of severe CDI. Furthermore, ribotypes 002 and 017 are both virulent strains with high antimicrobial resistance $(8,10,12)$, which may have been positively selected in the past because of excessive antimicrobial drug use. Now that the use of antimicrobial drugs has been declining, these strains may have seen a decline in prevalence, which lowered mortality rates. However, more data, such as those gathered through molecular typing and antibiotic resistance analysis, are required to validate this hypothesis.

Despite changes in CDI incidence, the proportion of CA-CDI cases of all CDI cases annually has remained steady from $2006-2019$, at $\approx 4-5 \%$. Risk factors for CA-CDI are unclear, although our data suggest that gastric acid suppression, antimicrobial drug use, and old age may be potential factors. Nonetheless, CA-CDI patients tend to be relatively younger. Previous studies have indicated that there is an increase in CA-CDI incidence and severe outcomes $(5,23)$. Our study, however, indicates the proportion of CA-CDI has remained relatively constant and that their clinical outcomes are generally more favorable, including lower rates of mortality and recurrence, than outcomes for HA-CDI patients. Furthermore, we did not observe any significant trend of increase in CA-CDI cases. One potential explanation is that Hong Kong still has lower rates of inflammatory bowel disease compared with Western countries, despite an increasing trend in these illnesses over the past few decades $(22,24)$.

The main strength of this study is that it is territorywide, driven by data extracted from all public hospitals in Hong Kong. All data, including demographics, laboratory results, drug prescription data, and procedures, were extracted from the public hospital database, thus reducing the possibility of recall bias. Furthermore, this study followed on our previous study (13) that investigated the epidemiology of CDI in Hong Kong during 2006-2014, providing a comprehensive epidemiologic pattern and comparisons for CDI in Hong Kong. Nonetheless, we acknowledge several limitations in our study. First, the data in this study were based only on 
inpatient data; we may have missed diagnoses made in outpatient clinics. Patients with mild symptoms could be sufficiently treated in outpatient clinics, which may result in an underestimation of the actual CDI incidence. Second, patient exposure to antimicrobial drugs and other drugs within 8 weeks of CDI was indicated as a logical indicator (i.e., true or false), regardless of dose, frequency, and prescription time, which may overestimate or underestimate the extent of the exposure. Third, there was a lack of data regarding changes in CDI diagnostic tests used from before 2015, making comparisons of CDI incidence unable to account for any shifts toward the use of the nucleic acid amplification test (NAAT), which is known to be a more sensitive test for CDI (2). Fortunately, the use of NAAT from 2015-2019 has remained within 40\%-50\%, which may mean a smaller impact on the trend of CDI incidence. Last, this is a retrospective study and there are unforeseeable covariates that were not adjustable or measurable, which may affect the analyses and results. Nevertheless, the epidemiology of CDI is dynamic, and changes can occur rapidly. We recommend continued surveillance of this infection in healthcare settings.

This project is supported by the Hong Kong University Grants Committee Research Grant Council (Early Career Scheme no. 21153816/24103516) and the Hong Kong Food and Health Bureau Commissioned Health and Medical Research Fund (CID-CUHK-C).

Author contributions: S.H.W. and W.K.K.W. conceived the study. C.L.T.G. and T.N.Y.K. extracted the data, performed the analysis, and drafted the manuscript. J.W.Y.M., L.Z., G.C.Y.L., G.L.H.W., M.I., S.S.L., J.Y., and J.J.Y.S. provided important intellectual input and revised the manuscript. J.J.Y.S., W.K.K.W., and S.H.W. managed and oversaw the study. All authors critically reviewed and agreed to be accountable for the works.

\section{About the Authors}

Mr. Guo is a medical student at the Chinese University of Hong Kong. Dr. Kwong is a postdoctoral researcher at the Chinese University of Hong Kong; his research interests include infectious diseases epidemiology and specifically Clostridioides difficile.

\section{References}

1. Leffler DA, Lamont JT. Clostridium difficile infection. N Engl J Med. 2015;372:1539-48. https:/ / doi.org/10.1056/ NEJMra1403772

2. Guh AY, Mu Y, Winston LG, Johnston H, Olson D, Farley MM, et al.; Emerging Infections Program
Clostridioides difficile Infection Working Group. Trends in U.S. burden of Clostridioides difficile infection and outcomes. N Engl J Med. 2020;382:1320-30. https:/ / doi.org/10.1056/ NEJMoa1910215

3. Heimann SM, Cruz Aguilar MR, Mellinghof S, Vehreschild MJGT. Economic burden and cost-effective management of Clostridium difficile infections. Med Mal Infect. 2018;48:23-9. https:/ / doi.org/10.1016/ j.medmal.2017.10.010

4. Rizzardi K, Norén T, Aspevall O, Mäkitalo B, Toepfer M, Johansson $\AA$, et al. National surveillance for Clostridioides difficile infection, Sweden, 2009-2016. Emerg Infect Dis. 2018;24:1617-25. https://doi.org/10.3201/eid2409.171658

5. Ofori E, Ramai D, Dhawan M, Mustafa F, Gasperino J, Reddy M. Community-acquired Clostridium difficile: epidemiology, ribotype, risk factors, hospital and intensive care unit outcomes, and current and emerging therapies. J Hosp Infect. 2018;99:436-42. https:/ / doi.org/10.1016/ j.jhin.2018.01.015

6. Valiente E, Cairns MD, Wren BW. The Clostridium difficile PCR ribotype 027 lineage: a pathogen on the move. Clin Microbiol Infect. 2014;20:396-404. https:// doi.org/10.1111/ 1469-0691.12619

7. Collins DA, Sohn KM, Wu Y, Ouchi K, Ishii Y, Elliott B, et al.; Clostridioides difficile Asia-Pacific Study Group. Clostridioides difficile infection in the Asia-Pacific region. Emerg Microbes Infect. 2019;9:42-52. https:/ / doi.org/10.108 0/22221751.2019.1702480

8. Imwattana K, Knight DR, Kullin B, Collins DA, Putsathit P, Kiratisin P, et al. Clostridium difficile ribotype 017characterization, evolution and epidemiology of the dominant strain in Asia. Emerg Microbes Infect. 2019;8:796807. https:// doi.org/10.1080/22221751.2019.1621670

9. Collins DA, Riley TV. Clostridium difficile in Asia: opportunities for One Health management. Trop Med Infect Dis. 2018;4:E7. https://doi.org/10.3390/tropicalmed4010007

10. Kong KY, Kwong TNY, Chan H, Wong K, Wong SSY, Chaparala AP, et al. Biological characteristics associated with virulence in Clostridioides difficile ribotype 002 in Hong Kong. Emerg Microbes Infect. 2020;9:631-8. https:/ / doi.org/ 10.1080/22221751.2020.1739564

11. Wong SH, Ip M, Hawkey PM, Lo N, Hardy K, Manzoor S, et al. High morbidity and mortality of Clostridium difficile infection and its associations with ribotype 002 in Hong Kong. J Infect. 2016;73:115-22. https://doi.org/10.1016/j. jinf.2016.05.010

12. Chow VCY, Kwong TNY, So EWM, Ho YII, Wong SH, Lai RWM, et al. Surveillance of antibiotic resistance among common Clostridium difficile ribotypes in Hong Kong. Sci Rep. 2017;7:17218. https://doi.org/10.1038/s41598-017-17523-7

13. Ho J, Dai RZW, Kwong TNY, Wang X, Zhang L, Ip M, et al. Disease burden of Clostridium difficile infections in adults, Hong Kong, China, 2006-2014. Emerg Infect Dis. 2017;23:1671-9. https://doi.org/10.3201/eid2310.170797

14. Sundararajan V, Henderson T, Perry C, Muggivan A, Quan H, Ghali WA. New ICD-10 version of the Charlson comorbidity index predicted in-hospital mortality. J Clin Epidemiol. 2004;57:1288-94. https:/ / doi.org/10.1016/ j.jclinepi.2004.03.012

15. Cohen SH, Gerding DN, Johnson S, Kelly CP, Loo VG, McDonald LC, et al.; Society for Healthcare Epidemiology of America; Infectious Diseases Society of America. Clinical practice guidelines for Clostridium difficile infection in adults: 2010 update by the Society for Healthcare Epidemiology of America (SHEA) and the Infectious Diseases Society of America (IDSA). Infect Control Hosp 
Epidemiol. 2010;31:431-55. https://doi.org/10.1086/651706

16. Thelwall S, Nsonwu O, Rooney G, Wasti S, Anselmo J, Hope R; Public Health England. Clostridium difficile infection: mandatory surveillance 2017/18. London: Public Health England; 2018.

17. Wilcox MH. Clostridium difficile surveillance in England. Infectious Agents Surveillance Report. 2020;41:39-41.

18. US Centers for Disease Control and Prevention. 2017 annual report for the Emerging Infections Program for Clostridioides difficile infection. 2017 [cited 2021 Oct 15]. https:/ / www.cdc. gov/hai/eip/pdf/cdiff/2017-CDI-Report-H.pdf

19. Liao F, Li W, Gu W, Zhang W, Liu X, Fu X, et al. A retrospective study of community-acquired Clostridium difficile infection in southwest China. Sci Rep. 2018;8:3992. https:/ / doi.org/10.1038/s41598-018-21762-7

20. Tariq R, Cho J, Kapoor S, Orenstein R, Singh S, Pardi DS, et al. Low risk of primary Clostridium difficile infection with tetracyclines: a systematic review and metaanalysis. Clin Infect Dis. 2018;66:514-22. https:/ / doi.org/10.1093/ cid/cix833

21. Ho P, Wu T, Chao DV, Hung IF, Lui L, Lung DC, et al. Reducing bacterial resistance with IMPACT, 5 th edition,
Hong Kong. Hong Kong: Centre for Health ProtectionDepartment of Health; 2017.

22. Lui RN, Wong SH, Lau LHS, Chan TT, Cheung KCY, $\mathrm{Li}$ A, et al. Faecal microbiota transplantation for treatment of recurrent or refractory Clostridioides difficile infection in Hong Kong. Hong Kong Med J. 2019;25:178-82.

23. Gupta A, Khanna S. Community-acquired Clostridium difficile infection: an increasing public health threat. Infect Drug Resist. 2014;7:63-72.

24. Ng SC, Leung WK, Shi HY, Li MK, Leung CM, Ng CK, et al. Epidemiology of inflammatory bowel disease from 1981 to 2014: results from a territory-wide population-based registry in Hong Kong. Inflamm Bowel Dis. 2016;22:1954-60. https:// doi.org/10.1097/ MIB.0000000000000846

Address for correspondence: Sunny H. Wong, Lee Kong Chian School of Medicine, 11 Mandalay Rd, Nanyang Technological University, Singapore 308232, Singapore; email: sunny.wong@ntu.edu.sg

\section{Emerging Infectious Diseases Spotlight Topics}
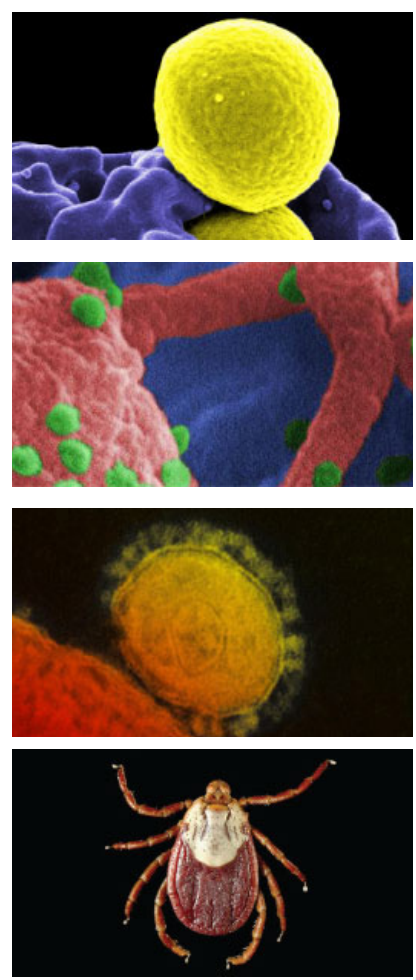

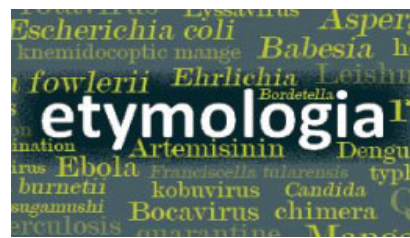

Antimicrobial resistance • Ebola Etymologia • Food safety • HIV-AIDS Influenza • Lyme disease • Malaria MERS • Pneumonia • Rabies • Ticks Tuberculosis • Coronavirus • Zika
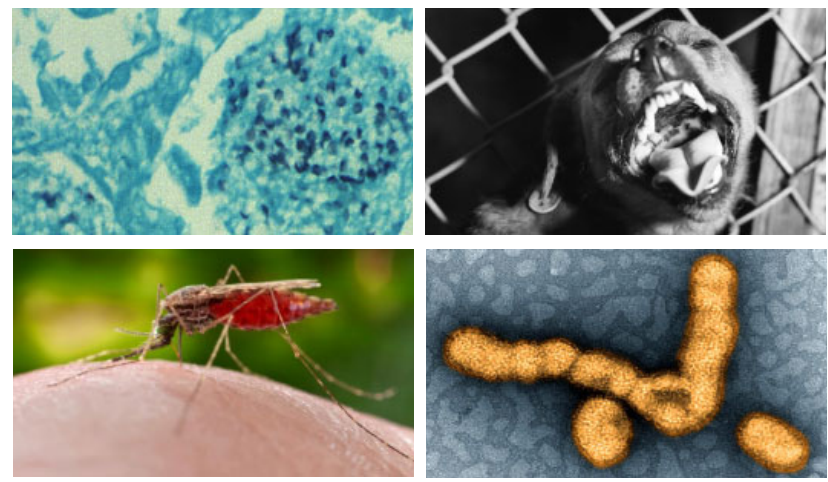
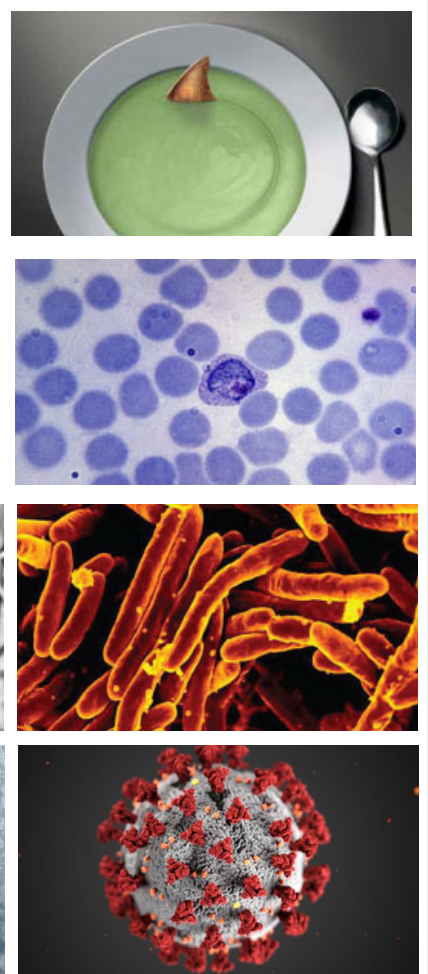

EID's spotlight topics highlight the latest articles and information on emerging infectious disease topics in our global community

\section{https://wwwnc.cdc.gov/eid/page/spotlight-topics}

